\title{
Finite-Temperature Collective Dynamics of a Fermi Gas in the BEC-BCS Crossover
}

\author{
M. J. Wright, ${ }^{1}$ S. Riedl, ${ }^{1,2}$ A. Altmeyer, ${ }^{1,2}$ C. Kohstall, ${ }^{1}$ E. R. Sánchez Guajardo, ${ }^{1}$ \\ J. Hecker Denschlag, ${ }^{1}$ and R. Grimm ${ }^{1,2}$ \\ ${ }^{1}$ Institut für Experimentalphysik und Forschungszentrum für Quantenphysik, Universität Innsbruck, 6020 Innsbruck, Austria \\ ${ }^{2}$ Institut für Quantenoptik und Quanteninformation, \\ Österreichische Akademie der Wissenschaften, 6020 Innsbruck, Austria
}

(Dated: October 26, 2018)

\begin{abstract}
We report on experimental studies on the collective behavior of a strongly interacting Fermi gas with tunable interactions and variable temperature. A scissors mode excitation in an elliptical trap is used to characterize the dynamics of the quantum gas in terms of hydrodynamic or nearcollisionless behavior. We obtain a crossover phase diagram for collisional properties, showing a large region where a non-superfluid strongly interacting gas shows hydrodynamic behavior. In a narrow interaction regime on the BCS side of the crossover, we find a novel temperature-dependent damping peak, suggesting a relation to the superfluid phase transition.

PACS numbers: 34.50.-s, 05.30.Fk, 39.25.+k, 32.80.Pj
\end{abstract}

Ultracold Fermi gases with tunable interactions have opened up intriguing possibilities to study the crossover from bosonic to fermionic behavior in strongly interacting many-body quantum systems [1]. In the zerotemperature limit, a Bose-Einstein condensate (BEC) of molecules is smoothly connected with a superfluid of paired fermions in the Bardeen-Cooper-Schrieffer (BCS) regime. In recent years, great progress has been achieved in the theoretical description of the ground state at zero temperature, and fundamental properties have been experimentally tested with considerable accuracy [2]. Finite-temperature phenomena in the BEC-BCS crossover, however, pose great challenges for their theoretical description. Experimental observations of finitetemperature behavior in the crossover have focussed on the measurement of condensate fractions [3, 4], on the spectroscopic investigation of pairing phenomena [5], or on the special case of unitarity-limited interactions $[6,7,[8]$.

To understand the collective dynamics of an ultracold quantum gas, it is crucial to study the conditions for hydrodynamic behavior. Collective mode experiments have probed the dynamics of strongly interacting Fermi gases for variable interaction strength near zero temperature [9, 10, 11, 12]. The results show the existence of both a hydrodynamic regime of collective motion and a near-collisionless regime with independent motion of the trapped particles. The role of temperature, however, remained essentially unexplored.

In this Letter, we explore the collective behavior of a finite-temperature, strongly interacting Fermi gas of ${ }^{6} \mathrm{Li}$ atoms throughout the BEC-BCS crossover. In order to characterize the transition from hydrodynamic to collisionless behavior, we analyze scissors mode oscillations at different temperatures. With varying temperature, the oscillations show a smooth transition between the two collisional regimes along with a broad maximum in the damping rate. We discover an unexpected sec- ond peak in the damping rate at lower temperatures in a narrow region on the BCS side of the crossover, where the gas remains hydrodynamic. This suggests the lowertemperature damping peak to be connected to the transition from a superfluid to a normal hydrodynamic gas.

The scissors mode in ultracold quantum gas experiments [13, 14] is an angular oscillation of the cloud about a principle axis of an elliptical trap, see Fig. 1(a). In our experiments, we confine the atoms in a harmonic, triaxial optical dipole trap. We choose the geometry of the trap to produce an elliptically shaped gas in the $\mathrm{x}-\mathrm{y}$ plane with very weak confinement along the $\mathrm{z}$ axis. The scissors mode experiments are done in the $\mathrm{x}-\mathrm{y}$ plane. In terms of trap frequencies, the standard configuration is $\omega_{x}>\omega_{y} \gg \omega_{z}$, where these trap frequencies are defined along the principle axes of the trap. If the gas is hydrodynamic, the angle of the gas oscillates collectively with a single frequency of $\left(\omega_{x}^{2}+\omega_{y}^{2}\right)^{1 / 2}$. If the gas is collisionless, the trapped atoms oscillate independently, resulting in a two-frequency oscillation. The larger frequency is given by $\omega_{x}+\omega_{y}$. When the collisional regime is changed this frequency is adiabatically connected to the hydrodynamic frequency. The smaller frequency is given by $\omega_{x}-\omega_{y}$ and is absent in the hydrodynamic limit [13].

The preparation of a strongly interacting Fermi gas of ${ }^{6} \mathrm{Li}$ proceeds in the same way as described in our previous work [12, 15]. The result is a deeply degenerate, balanced two-component spin mixture of typically $N=4 \times 10^{5}$ atoms with tunable $s$-wave interactions near a broad Feshbach resonance, which is centered at a magnetic field $B=834 \mathrm{G}$. Rapid spatial modulation of the trapping beam by two acousto-optical deflectors is used to create a time-averaged elliptical trapping potential for the scissors mode [12]. The aspect ratio is set to $\omega_{x} / \omega_{y} \approx 2.0$. We employ a trap with frequencies $\omega_{x}=2 \pi \times 830 \mathrm{~Hz}$ and $\omega_{y}=2 \pi \times 415 \mathrm{~Hz}\left(\omega_{z}=\right.$ $2 \pi \times 22 \mathrm{~Hz}$ ), if not indicated otherwise. This results in a Fermi temperature $T_{F}=\left(\hbar \bar{\omega} / k_{B}\right)(3 N)^{1 / 3}=0.94 \mu \mathrm{K}$, 
(a)
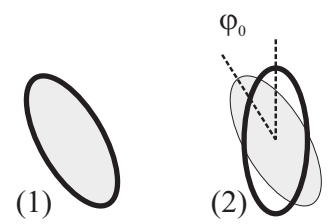

(3)

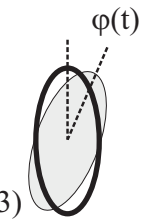

(b)
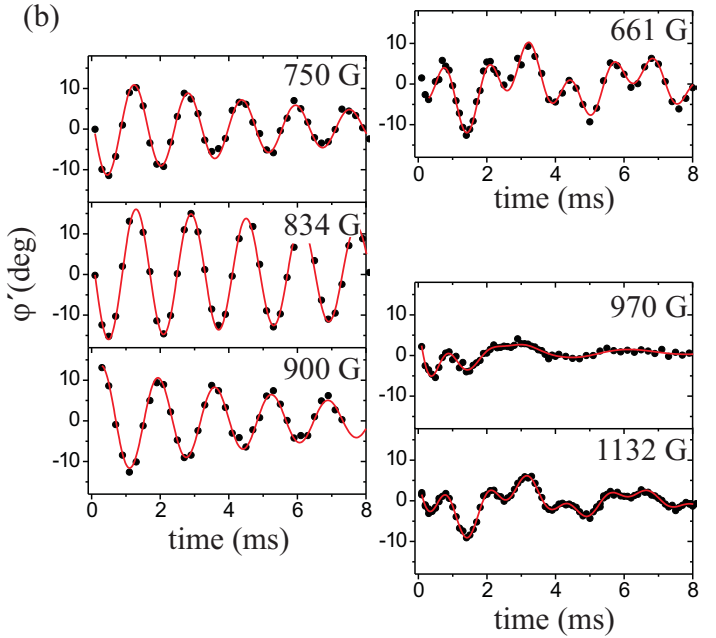

Figure 1: (a) Schematic showing the excitation of the scissors mode. (1) The gas (shaded region) is at rest, in equilibrium with the trap (heavy solid line). (2) The trap is suddenly rotated. (3) The gas oscillates around the new equilibrium position. (b) Scissors mode oscillations observed at the lowest obtainable temperature $\left(T \approx 0.1 T_{F}\right.$ at $\left.834 \mathrm{G}\right)$ for various magnetic fields. On the left side, where $B=750 \mathrm{G}, 834 \mathrm{G}$, and $900 \mathrm{G}\left(1 / k_{F} a=1.4,0.0\right.$, and -0.6$)$, the gas is hydrodynamic. On the right side, where $B=661 \mathrm{G}, 970 \mathrm{G}$, and $1132 \mathrm{G}$ $\left(1 / k_{F} a=5.0,-1.0\right.$, and -1.44$)$, the gas is nearly collisionless and exhibits the characteristic two-frequency oscillation. Here $\omega_{x}=2 \pi \times 580 \mathrm{~Hz}, \omega_{y}=2 \pi \times 270 \mathrm{~Hz}$, and $T_{F}=0.69 \mu \mathrm{K}$.

where $\bar{\omega}=\left(\omega_{x} \omega_{y} \omega_{z}\right)^{1 / 3}$. The trap depth corresponds to about $12 T_{F}$. To excite the scissors mode, we suddenly rotate the angle of the trap by $\sim 5$ degrees, see Fig. 1(a).

The angle of the oscillating cloud is determined by processing absorption images, taken after a short expansion time of $400 \mu \mathrm{s}$. A two-dimensional Thomas-Fermi profile is fit to the images, where the tilt of the principle axes of the cloud is a free parameter, see Fig. 1(a). Note that the short expansion somewhat decreases the ellipticity of the cloud, but increases the amplitude of the scissors mode oscillation [16]. In the hydrodynamic regime, we fit a damped cosine function to the experimental data. In the collisionless regime, we fit the oscillation to a sum of two damped cosine functions each with their own free parameters. In the region between these two limits, we find that a single damped cosine function fits the data reasonably well, as the lower-frequency component damps out very quickly [13].

First, we examine the collective behavior of the gas at our lowest obtainable temperatures. We compare scis-

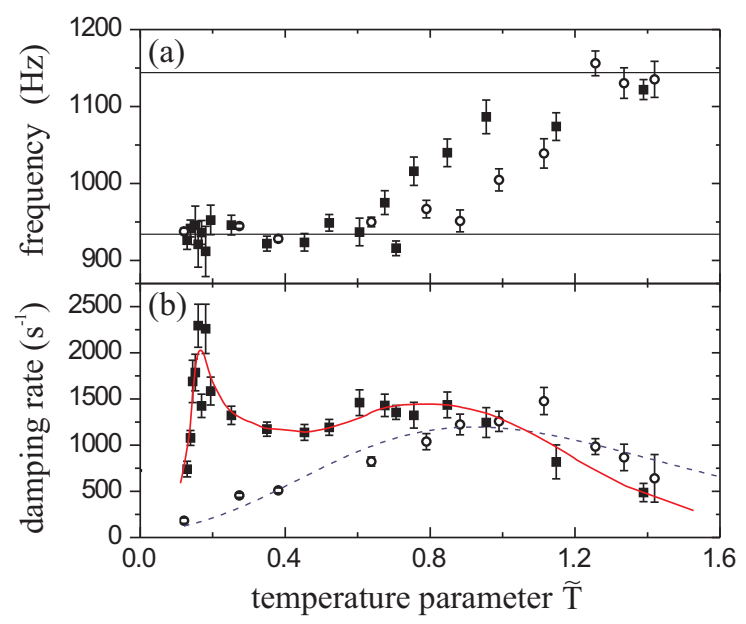

Figure 2: Frequency and damping rate for the scissors mode oscillation for $B=895 \mathrm{G}\left(1 / k_{F} a=-0.45\right.$, solid squares $)$ and at $B=834 \mathrm{G}\left(1 / k_{F} a=0\right.$, open circles $)$. The frequency limits in the hydrodynamic and collisionless regimes are shown by the horizontal lines in (a), including small corrections for the anharmonicity of the trap [17]. The lines in (b) are introduced as guides to the eye. For $\tilde{T}$ greater than 1.14, the scissors mode oscillations are fit by a two-frequency cosine function (for details see text).

sors mode oscillations at different settings of the magnetic field, i.e. different values of $1 / k_{F} a$. Typical scissors mode oscillations are shown in Fig. 1(b). At $B=661$ $\mathrm{G}$, far on the BEC side of resonance, the gas exhibits nearly collisionless behavior. Here inelastic collisions result in heating the gas above the critical temperature for BEC. In the regime where the gas is strongly interacting, $B=750 \mathrm{G}, 834 \mathrm{G}$, and $900 \mathrm{G}$, the gas oscillates collectively. High precision measurements taken at $B=834$ $G$ show the scissors mode oscillation yields a frequency that agrees with theory within one percent. Far on the BCS side, at $B=970 \mathrm{G}$ and $1132 \mathrm{G}$, the gas exhibits behavior that is nearly collisionless. The abrupt transition between the hydrodynamic and collisionless regimes at low temperature occurs at essentially the same magnetic field, $B \approx 950 \mathrm{G}$, as in other collective mode experiments [9, 10, 12].

To explore finite-temperature collisional behavior, we extend the scissors mode measurements. To set the temperature, we use a controlled heating scheme. Here, we suddenly compress the trap and allow for subsequent equilibration [18]. We control the temperature of the gas by adjusting the amount of compression.

The determination of the temperature $T$ in an ultracold, strongly interacting Fermi gas is in general difficult [19]. We can measure an effective temperature (or entropy) parameter $\tilde{T}$ at the center of the Feshbach resonance, $B=834 \mathrm{G}$. We determine $\tilde{T}$ by fitting integrated, one-dimensional, density profiles in the manner described in [19, 20, 21]. At $B=834 \mathrm{G}$, for $T / T_{F}>0.3$, the pa- 


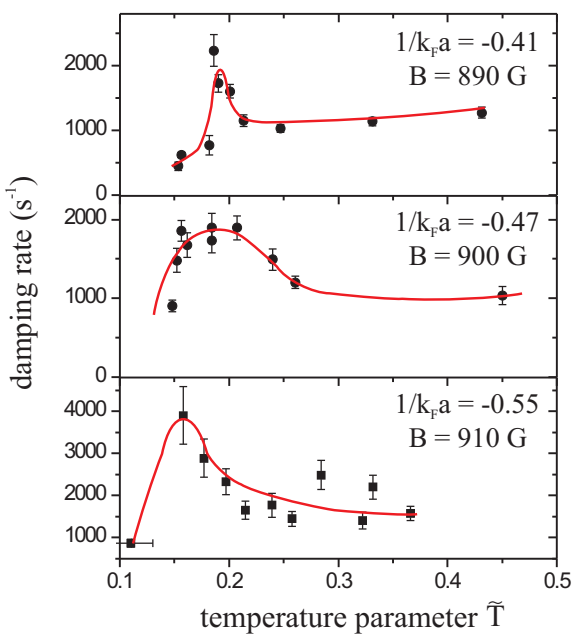

Figure 3: Low-temperature damping peak observed in a narrow magnetic-field region at the BCS side of the resonance $\left(1 / k_{F} a \approx-0.5\right)$. The solid lines are introduced as guides to the eye.

rameter $\tilde{T}$ is proportional to the real temperature with $T / T_{F} \approx \tilde{T} / 1.5$. For lower temperatures, an empirical conversion has been determined [19, 20, 21, 22]. The parameter $\tilde{T}$, measured in the unitarity limit at $834 \mathrm{G}$, can be used also as a temperature scale for other interaction regimes under the condition that entropy is conserved in adiabatic sweeps of the magnetic field [23].

In Figure 2, we show the frequency and damping rate as a function of $\tilde{T}$ for two cases, in the unitarity limit $\left(1 / k_{F} a=0.00\right)$ and at the BCS side of the crossover $\left(1 / k_{F} a=-0.45\right)$. The frequency behavior in Fig. 2(a) is qualitatively the same for both cases. At low temperatures, the gas shows the hydrodynamic frequency and, at the highest temperatures, we observe the behavior characteristic for the collisionless gas. With varying temperature, the changing frequency smoothly connects the hydrodynamic with the collisionless regime. Quantitatively, the transition occurs at somewhat higher $\tilde{T}$ in the unitarity limit. In the transition region, the damping rate shows a maximum that accompanies the change in frequency, see Fig. 2(b). We introduce the temperature parameter $\tilde{T}_{H}$ for this damping maximum, marking the transition between hydrodynamic and collisionless behavior.

The temperature dependence of the damping rate in Fig. 2(b) reveals a qualitatively different behavior between the two interaction regimes. An additional peak shows up at lower temperatures for the BCS side of the crossover, while this peak is absent in the unitarity limit. Remarkably, this novel feature is not associated with a change in the frequency.

We could detect the low-temperature damping peak only in a very narrow range at the BCS side of the crossover. This feature was found between magnetic

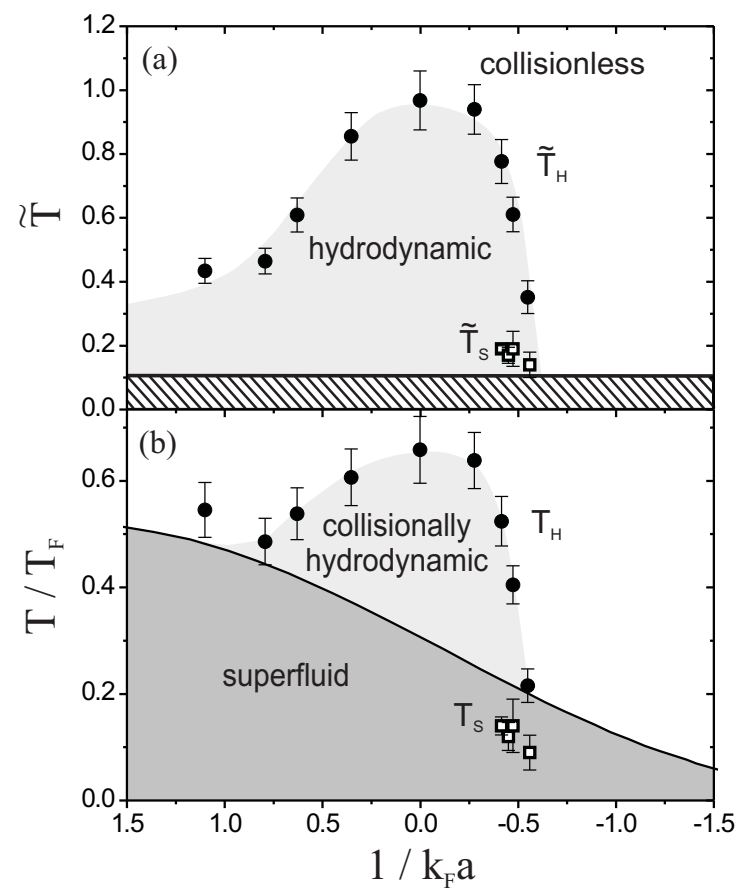

Figure 4: Phase diagram for the hydrodynamic behavior of the scissors mode in terms of (a) the temperature parameter $\tilde{T}$ and (b) the real temperature $T$. The smooth transition from hydrodynamic to collisionless is characterized by the temperature parameter $\tilde{T}_{H}$ (temperature $T_{H}$ ). The second damping peak near $1 / k_{F} a \approx-0.5$ is marked by $\tilde{T}_{S}\left(T_{S}\right)$. In (a) the hatched region indicates the region $(\tilde{T}<0.1)$ where our thermometry does not produce reliable results. In (b) the solid line shows a theoretical curve for the phase transition to superfluidity [24].

fields of $890 \mathrm{G}$ and $920 \mathrm{G}$, corresponding to interaction parameters $1 / k_{F} a$ between -0.6 and -0.4 . In Fig. 3, we show the low-temperature damping peak as it changes in this narrow region. Closer to resonance, the peak becomes very narrow, shifts toward higher temperatures, and finally seems to disappear. To mark the location of this peak, we introduce the temperature parameter $\tilde{T}_{S}$.

We now discuss our observations in terms of a crossover phase diagram for the scissors mode excitation [25]. In Fig. 4(a), we plot $\tilde{T}_{H}$ (closed circles) and $\tilde{T}_{S}$ (open squares) as a function of the interaction parameter. The data points for $\tilde{T}_{H}$ show a pronounced maximum at the center of the resonance. To facilitate an interpretation of the experimental data, we convert $\tilde{T}_{H}$ and $\tilde{T}_{S}$ into real temperatures $T_{H}$ and $T_{S}$, following the theory of Ref. 23]. Fig. 4(b) shows the resulting phase diagram, including a theoretical prediction 24] of the temperature $T_{C}$ for the phase transition to a superfluid state.

Near the center of the Feshbach resonance, hydrodynamic behavior is observed far above the superfluid transition temperature. The large difference between $T_{H}$ and $T_{C}$ confirms the existence of a non-superfluid hydrodynamic region above $T_{C}[\underline{6}, \underline{8}, 26]$. Our measurements 
show that this normal-gas hydrodynamic regime is restricted to the narrow, strongly interacting region near resonance where $1 / k_{F} a$ stays well below unity. On the BEC side, $T_{H}$ is close to the expected value for $T_{C}$. Here one can assume that hydrodynamic behavior essentially results from the formation of a molecular BEC. A surrounding non-condensed molecular gas would exhibit near-collisionless properties, similar to what has been measured in atomic BEC experiments [27]. On the BCS side of resonance, $T_{H}$ falls off very rapidly. In this region, collective modes may couple to the weakly bound fermion pairs [9, 12]. We did not observe hydrodynamic behavior beyond that point.

For the low-temperature damping peak found at the BCS side of the crossover near $1 / k_{F} a \approx-0.5$, our phase diagram in Fig. 4(b) suggests a close relation to the superfluid phase transition. The peak occurs at roughly $0.6 T_{C}$, and it follows the general behavior of the superfluid transition to move toward higher temperature as it approaches the resonance. This points to a scenario where a substantial superfluid core in the center of the trap is surrounded by a non-superfluid, but still hydrodynamic fraction in the outer region of the trap. Whether damping results from the coupling of these two components or whether other mechanisms are responsible for this phenomenon remains an open question. We note that the low-temperature damping peak is not specific to the scissors mode. We have also found a corresponding, but less pronounced peak in measurements of the radial breathing mode. Further investigations and better theoretical understanding will be required to answer the intriguing question whether the novel damping peak does indeed mark the transition from the normal hydrodynamic to the superfluid state.

In conclusion, we have investigated hydrodynamic behavior at finite temperatures in the BEC-BCS crossover using scissors mode excitations. Our measurements highlight the existence of a region of non-superfluid hydrodynamics in the strongly interacting regime where $\left|k_{F} a\right| \gtrsim 1$. In the unitarity limit, predominant hydrodynamic behavior is found up to $\sim 0.6 T_{F}$, which substantially exceeds the superfluid transition temperature of $\sim 0.3 T_{F}$. With increasing temperature, the transition from hydrodynamic to collisionless behavior proceeds in general smoothly and is accompanied by a local maximum of damping. In addition, we have discovered a novel low-temperature damping peak at the BCS side of the crossover, which suggests a relation to the superfluid phase transition. With this observation, experiments on collective oscillation modes of Fermi gases in the BECBCS crossover continue to produce puzzling observations [6, 9, 12] with the potential to stimulate deeper theoretical understanding of the physics of strongly interacting Fermi gases.

We thank S. Stringari for stimulating discussions. We also thank Q. Chen, K. Levin, and J. E. Thomas for dis- cussions concerning determination of the temperature. We acknowledge support by the Austrian Science Fund (FWF) within SFB 15 (project part 21). M.J.W. is supported by a Marie Curie Incoming International Fellowship within the 6th European Community Framework Program.

[1] M. Inguscio, W. Ketterle, and C. Salomon, eds., Ultracold Fermi Gases (IOS Press, Amsterdam, 2007), Proceedings of the International School of Physics "Enrico Fermi", Course CLXIV, Varenna, 20-30 June 2006, in press.

[2] S. Giorgini, L. P. Pitaevskii, and S. Stringari, arXiv:0706.3360.

[3] C. A. Regal, M. Greiner, and D. S. Jin, Phys. Rev. Lett. 92, 040403 (2004).

[4] M. W. Zwierlein et al., Phys. Rev. Lett. 92, 120403 (2004).

[5] C. Chin et al., Science 305, 1128 (2004).

[6] J. Kinast, A. Turlapov, and J. E. Thomas, Phys. Rev. Lett. 94, 170404 (2005).

[7] J. T. Stewart, J. P. Gaebler, C. A. Regal, and D. S. Jin, Phys. Rev. Lett. 97, 220406 (2006).

[8] B. Clancy, L. Luo, and J. E. Thomas, arXiv:0705.2782.

[9] M. Bartenstein et al., Phys. Rev. Lett. 92, 203201 (2004).

[10] J. Kinast, A. Turlapov, and J. E. Thomas, Phys. Rev. A 70, 051401(R) (2004).

[11] A. Altmeyer et al., Phys. Rev. Lett. 98, 040401 (2007).

[12] A. Altmeyer et al., arXiv:0704.3366.

[13] D. Guéry-Odelin and S. Stringari, Phys. Rev. Lett. 83, 4452 (1999).

[14] O. M. Maragò et al., Phys. Rev. Lett. 84, 2056 (2000).

[15] S. Jochim et al., Science 302, 2101 (2003).

[16] M. Modugno et al., Phys. Rev. A 67, 023608 (2003).

[17] S. Riedl et al., to be published.

[18] Sudden compression of the trap excites the axial mode which is long lived. Since the frequency is much smaller than the scissors mode frequency, it can be neglected. Nonetheless, we carried out a direct comparison without the axial mode present and found the same behavior.

[19] J. Kinast et al., Science 307, 1296 (2005).

[20] J. Kinast, Ph.D. thesis, Duke University (2006).

[21] J. Stajic, Q. Chen, and K. Levin, Phys. Rev. Lett. 94, $060401(2005)$.

$[22] \tilde{T} \approx 1.2\left(T / T_{F}\right)^{1.49}$ for $\left(T / T_{F}\right)<0.3$.

[23] Q. Chen, J. Stajic, and K. Levin, Phys. Rev. Lett. 95, 260405 (2005).

[24] A. Perali, P. Pieri, L. Pisani, and G. C. Strinati, Phys. Rev. Lett. 92, 220404 (2004).

[25] In a comparative study of different collective modes [17] we found the scissors mode to behave very similar to the radial quadrupole mode [12], which is also a surface mode. The radial compression mode behaves quite differently [6, 17]. The axial mode [9] shows in general hydrodynamic behavior in a much wider parameter range because of its much lower frequency.

[26] A. Minguzzi and M. P. Tosi, Phys. Rev. A 63, 023609 (2001).

[27] O. Maragò, G. Hechenblaikner, E. Hodby, and C. Foot, Phys. Rev. Lett. 86, 3938 (2001). 